\title{
INVESTIGATION AND BIOLOGICAL CONTROL OF TOXIGENIC FUNGI AND MYCOTOXINS IN DAIRY CATTLE FEEDS
}

\section{ABSTRACT}

Mycotoxins, the secondary fungal metabolites are important contaminants of food and feed. Among the other contaminants, aflatoxin B1 (AFB1) and OTA are frequently detected in the animal feed product.

In the present study, the mixed dairy cow feed products were collected from the supermarkets in Qatar and analyzed for the presence of AFB1 and OTA. Yeast strains were isolated and tested for their biological control activities against aflatoxigenic and ochratoxin fungi. We demonstrated that local 15 yeasts isolates have important antifungal potential activities through the synthesis of Volatile Organic Compounds (VOC) that are able to act against the mycotoxigenic fungi and their synthesis of the mycotoxins. Two Yeast strains (4\&2) isolated from fermented food, have shown a great antifungal inhibition growth in-vitro as well as spores inhibition and mycotoxins synthesis.

\section{OBJECTIVES}

1. Monitoring the presence of fungi and mycotoxins in Dairy Cattle Feeds.

2. Isolation and Application of local yeasts strains volatile organic compounds in the biological control of toxigenic fungi in Qatar.

\section{LITERATURE REVIEW}

Mycotoxins are very large and diverse group of toxic secondary metabolites of low molecular weight. They are produced by filamentous fungi mainly belonging to the genera Aspergillus, Penicillium, Alternaria and Fusarium. In 2019, more than 399 mycotoxins have been investigated, identified and reported so far (Carolina Santos Pereira, 2019). The widely discovered mycotoxins are aflatoxins such as patulin, citrinin, ochratoxin (OTA), and trichothecene including Fumonisins, deoxynivalenol (DON), HT2 toxin (HT2), T2 poison (T2) and Zearalenone (ZEN) Aflatoxins are generally produced by different kind of Aspergillus species such as Aspergillus flavus and Aspergillus parasiticus. However, $A$. nomius \& $A$. pseudotamarri are very known to produce AFs.

\section{METHODOLOGY}

Part 1. Isolation and identification of Aflatoxigenic fungi from dairy animal feed samples:

Dairy animal feed samples were collected from animal feed market, located in Doha-Qatar.

Collected samples included cereal grains and cerealsmixed ration.

After dilution in sterile distilled water, samples were plate inoculated in Dichloran-Rose bengalChloramphenicol agar (selective media for fungi). Developing fungal colonies were transferred to identification media including malt extract agar (MEA), Czpeck yeast extract (CYA) agar and glycerol nitrate agar (G25N).

Part 2. Exploration of mycotoxin synthesis potential of fungi (in vitro):

The mycotoxins were extracted by adding a volume of $10 \mathrm{ml}$ of $80 \%$ methanol extraction solvent to the supernatant.

The mycotoxin extracts were filtered by $24 \mathrm{~cm}$ filter paper.

Mycotoxin concentration was determined using ELISA RIDA Screen ELISA kits.

Part 3. Isolation of Yeast strains from dairy products, meat products $\&$ fermented food:

Yeasts were isolated from the Laban and dairy products.

Meat \& dairy products were plated on selective media like YPDA \& YCA.

Part 4. Screening of yeasts for antifungal activity and molecular identification:

For the screening of antifungal activities of yeast species, co-culture method described by Farbo et al., 2018 was followed.

1- Caw feed samples contain different of Aflatoxin \& OTA concentrations

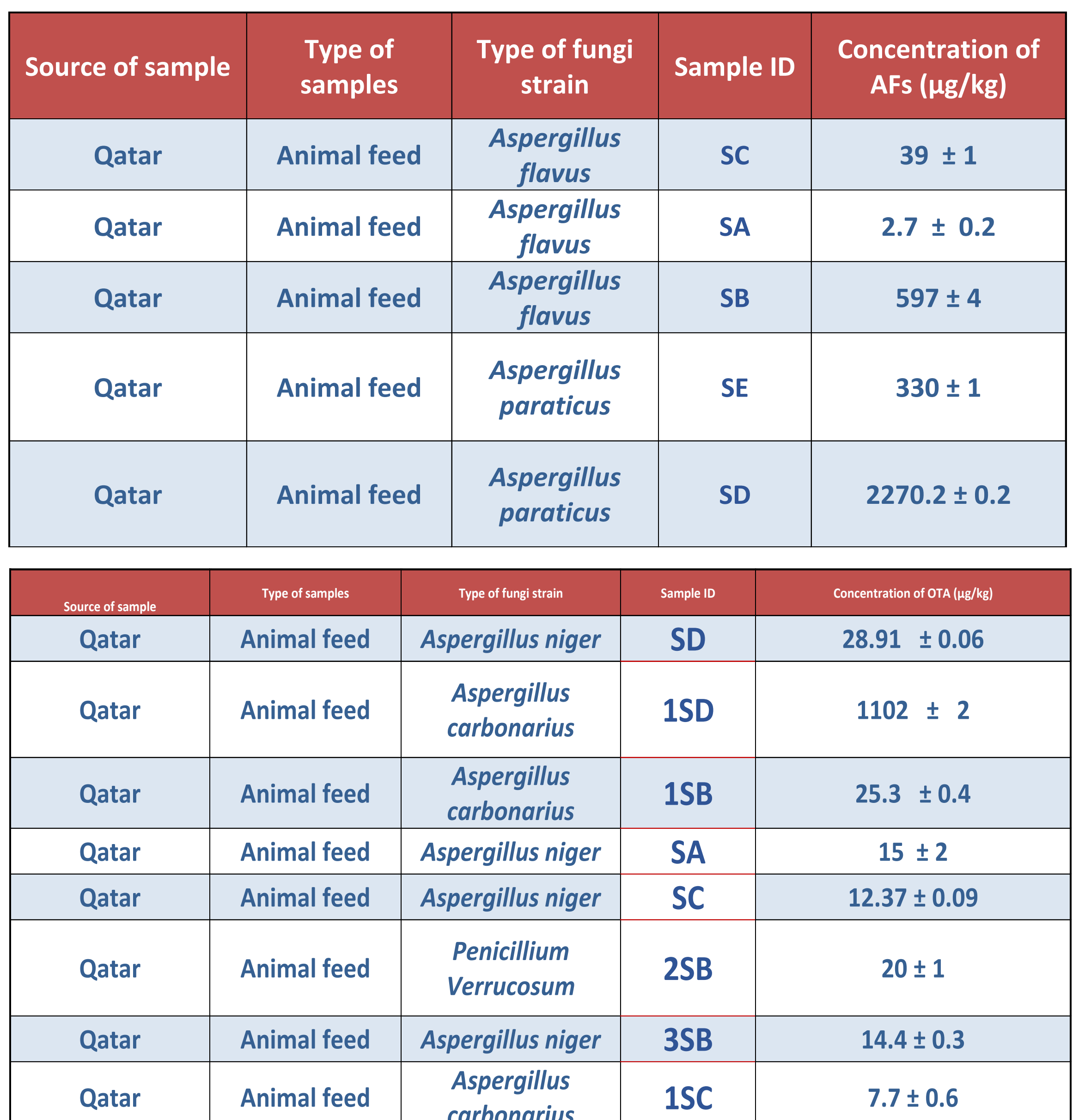

${ }^{1}$ Values in parenthesis indicate positive percentages; ${ }^{2} \mathrm{EU}$ maximum limits for AFs in animal feeds $20 \mathrm{ul} / \mathrm{kg}$, and for OTA $250 \mathrm{ug} / \mathrm{kg}$.

\section{REFERENCES}

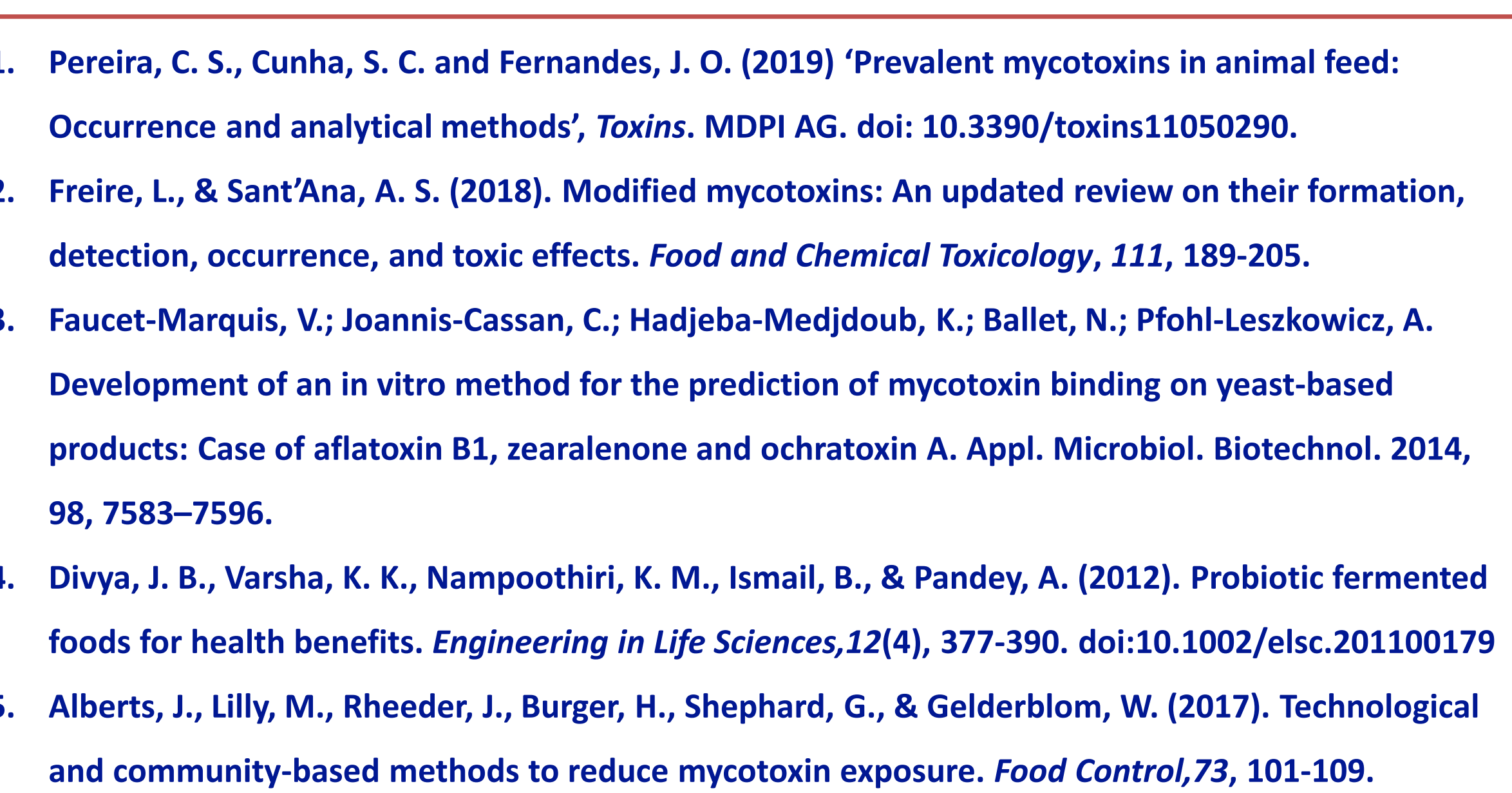

\section{RESULTS AND DISCUSSION}

2- Mycotoxigenic fungi were isolated and identified from mixed cow dairy feed :

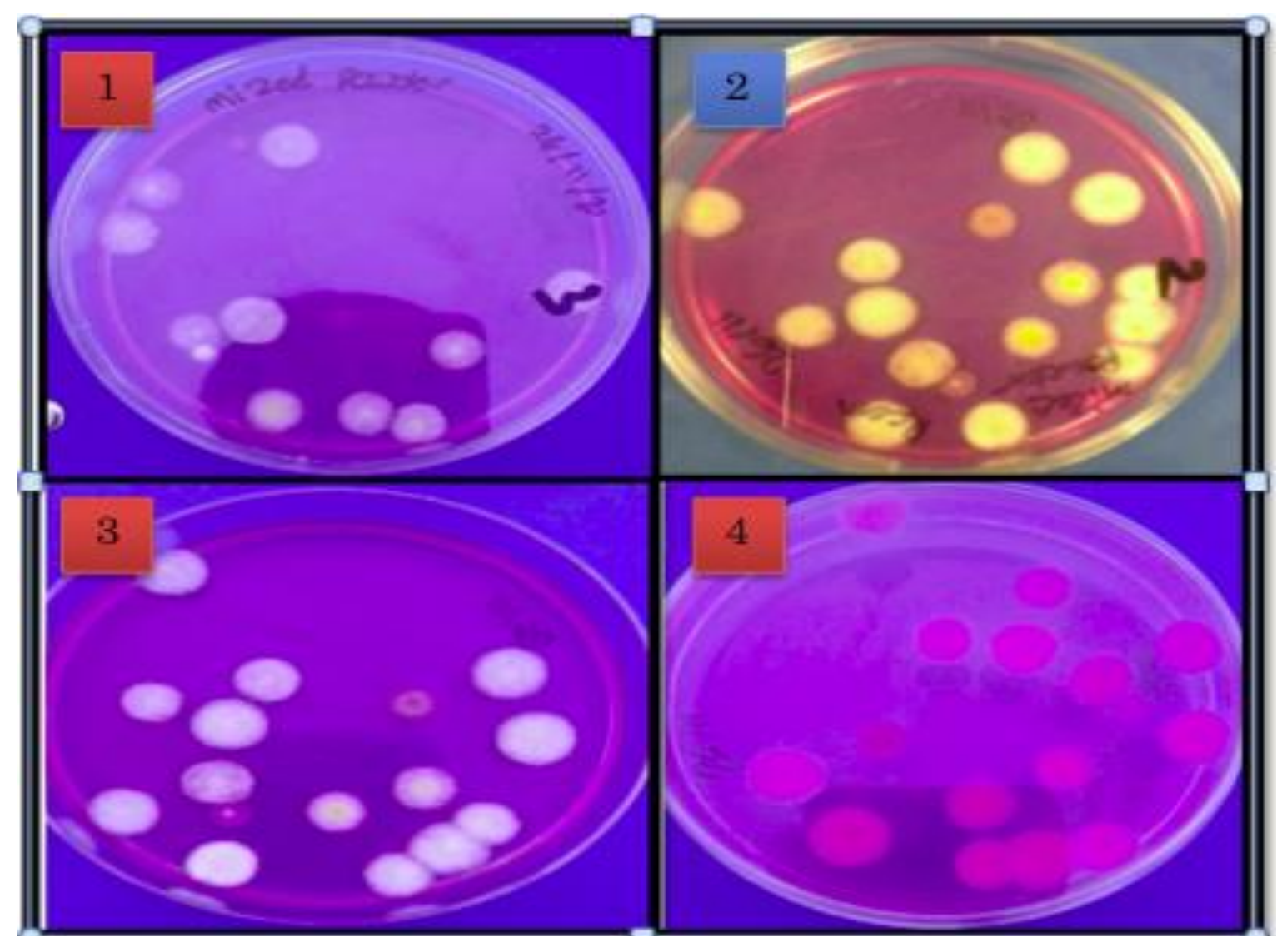

Fungal clones in Dichloran-Rose Bengal-Chloramphenicol agar. Aspergillus parasiticus isolated and identified from mixed cow dairy feed :

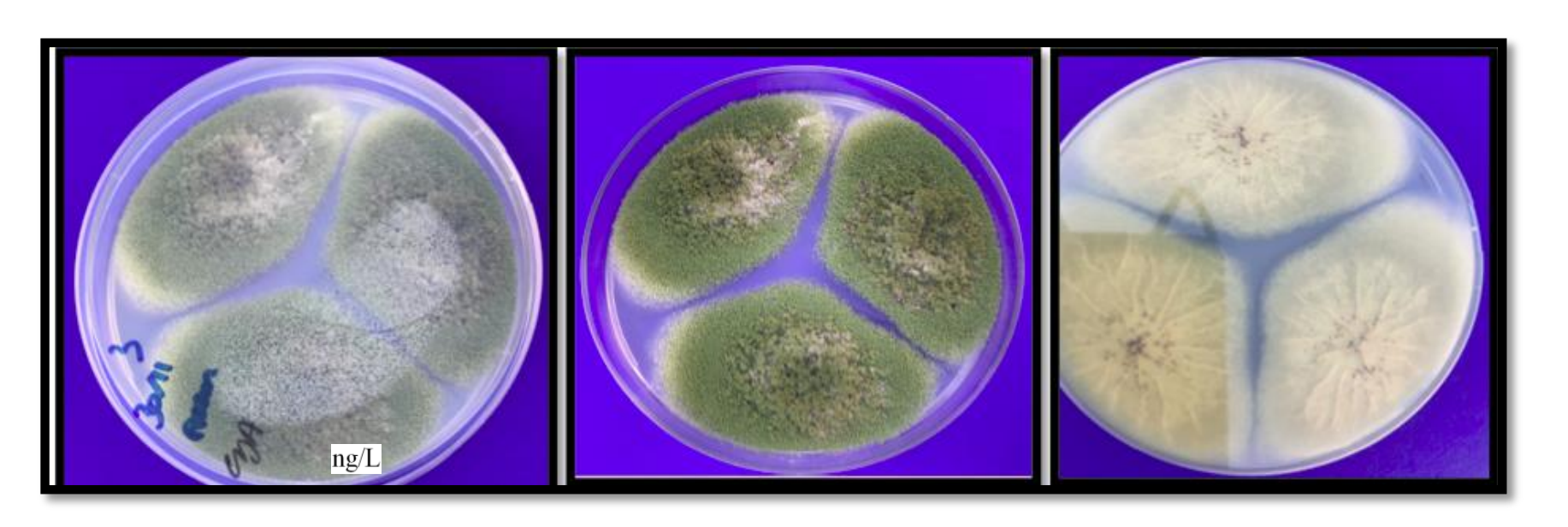

Aspergillus flavus isolated and identified on Malt Extract Agar

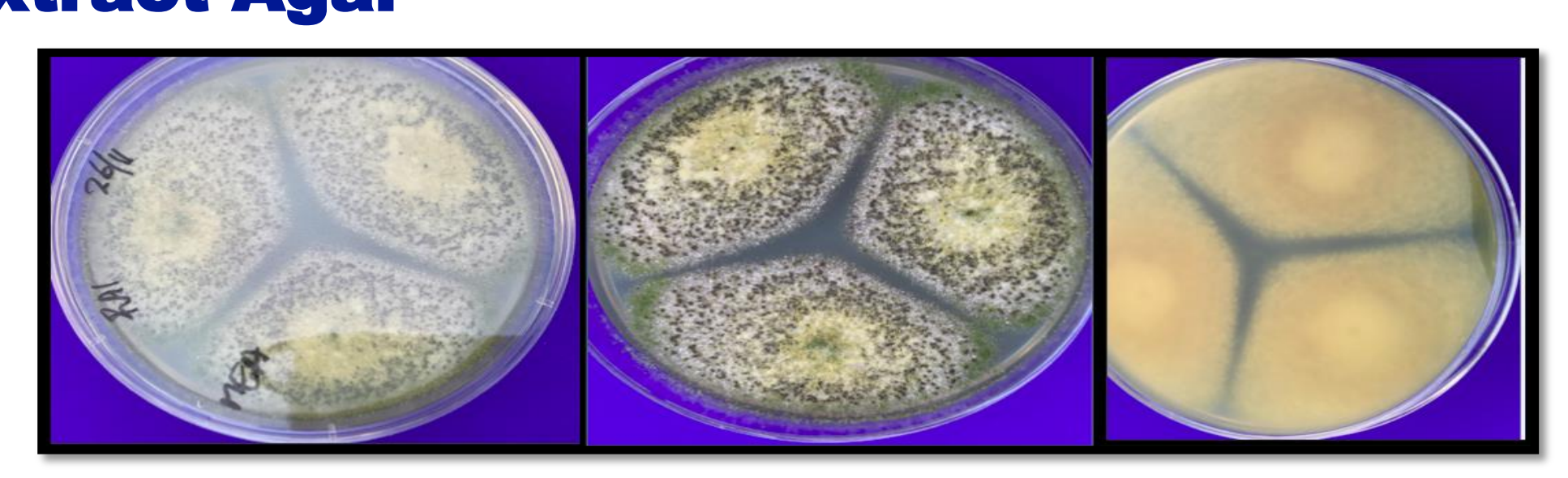

Aspergillus flavus isolated and identified Glycerol nitrate agar.

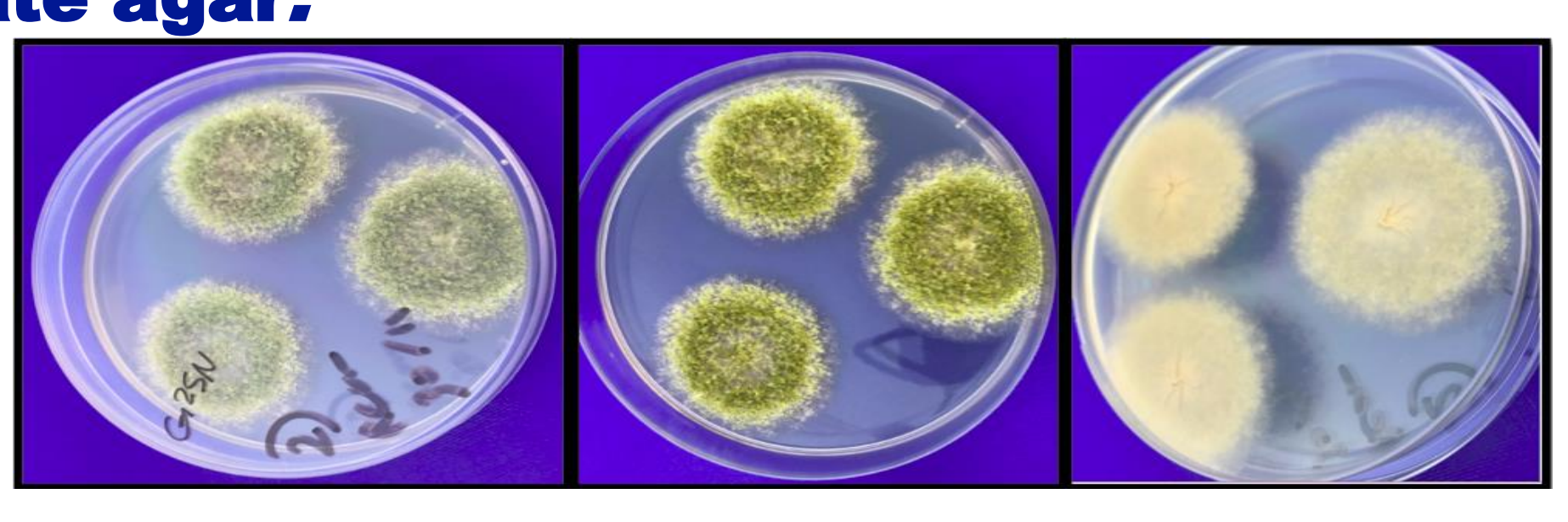

3. Local yeast strains were Isolated from dairy 3. Local yeast strains were Isolated from dairy
product samples on selective media like YPDA \& Product
YCA.

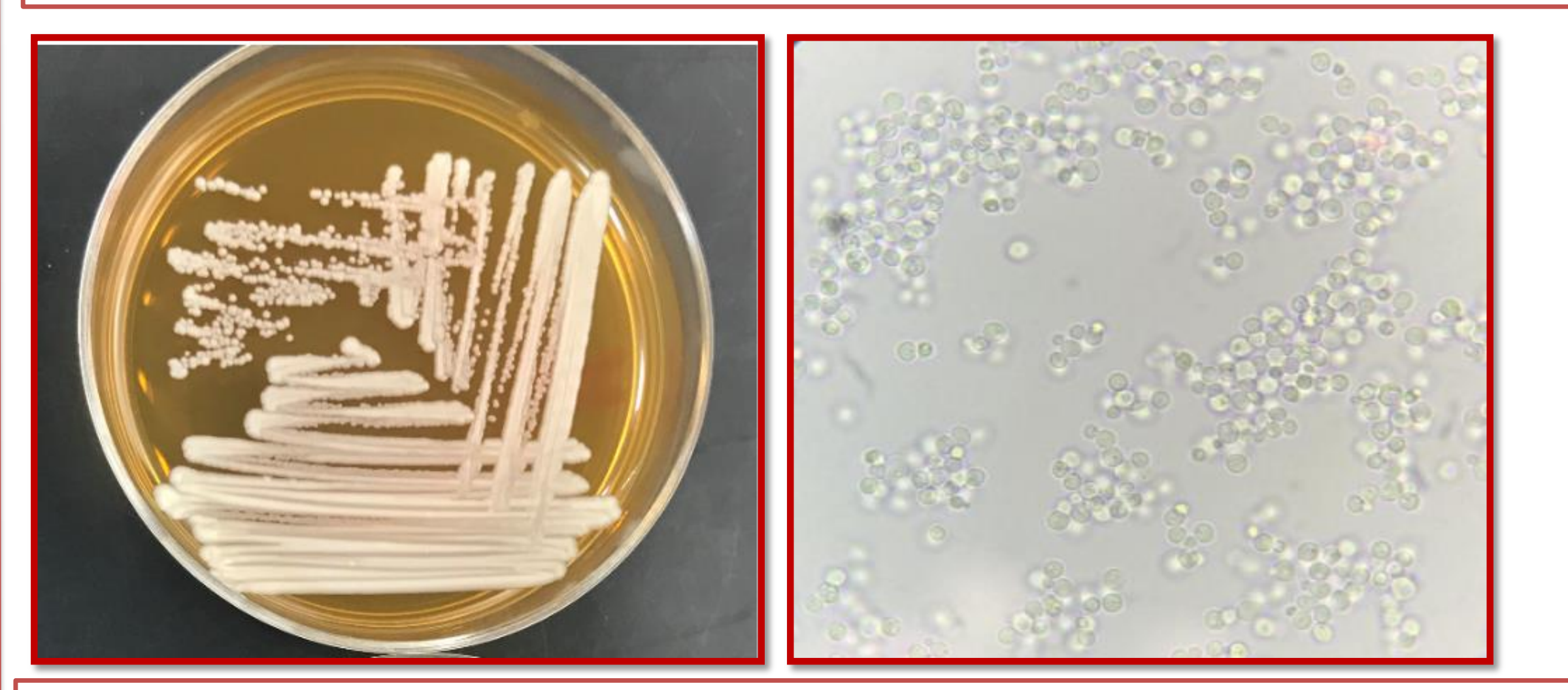

4. Screening of yeasts for VOC antifungal activities and molecular identification:

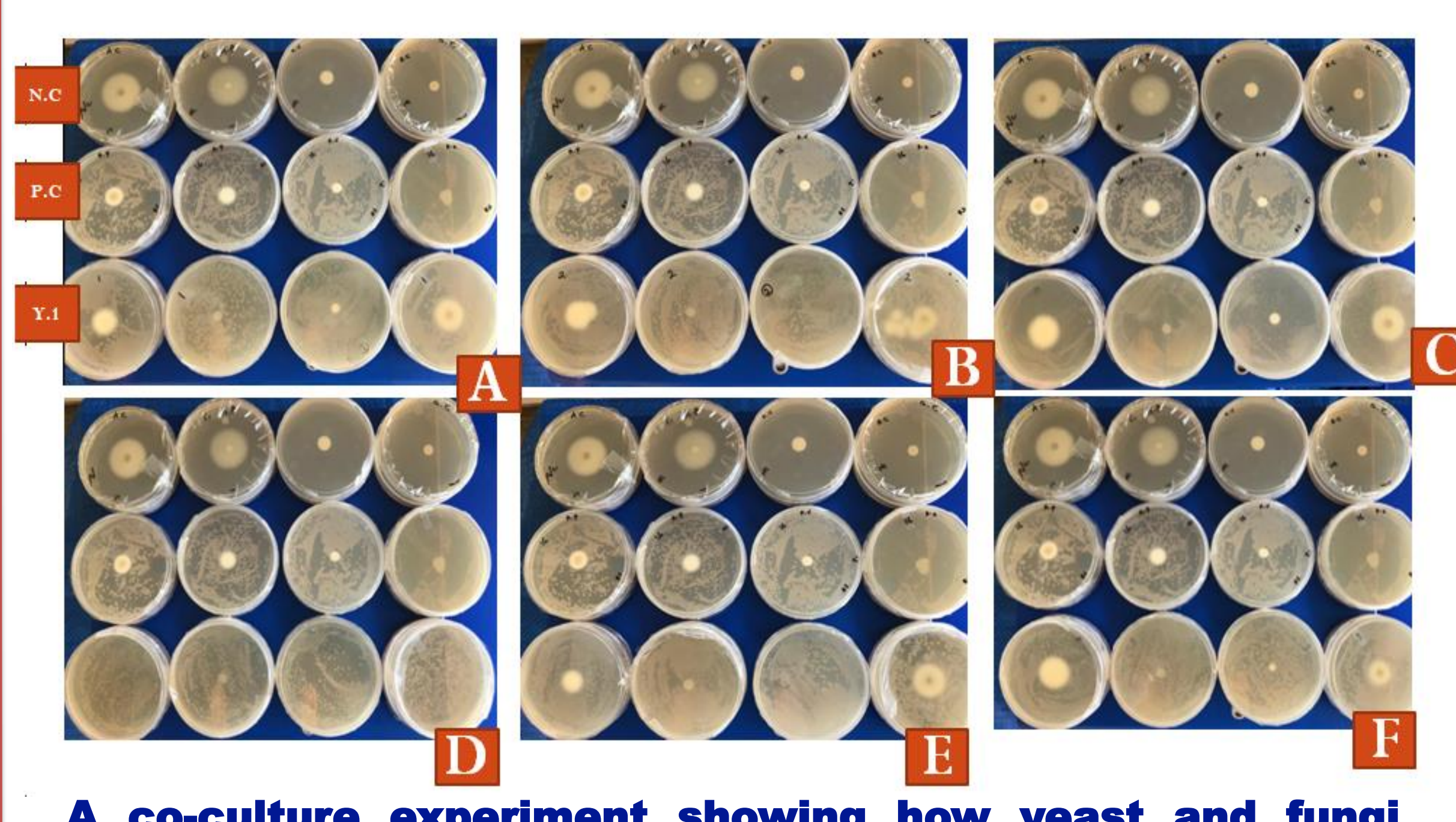

A co-culture experiment showing how yeast and fungi A co-culture experiment showing how yeast and fung
were sealed together, and after 4 days of incubation
found that significant differences in the yeast antifungal found that significant, differences in the yeast antifunga
activates (A) Y1, (B) Y2, (C) Y15, (D) Y4, (E) Y7, (F) Y3.

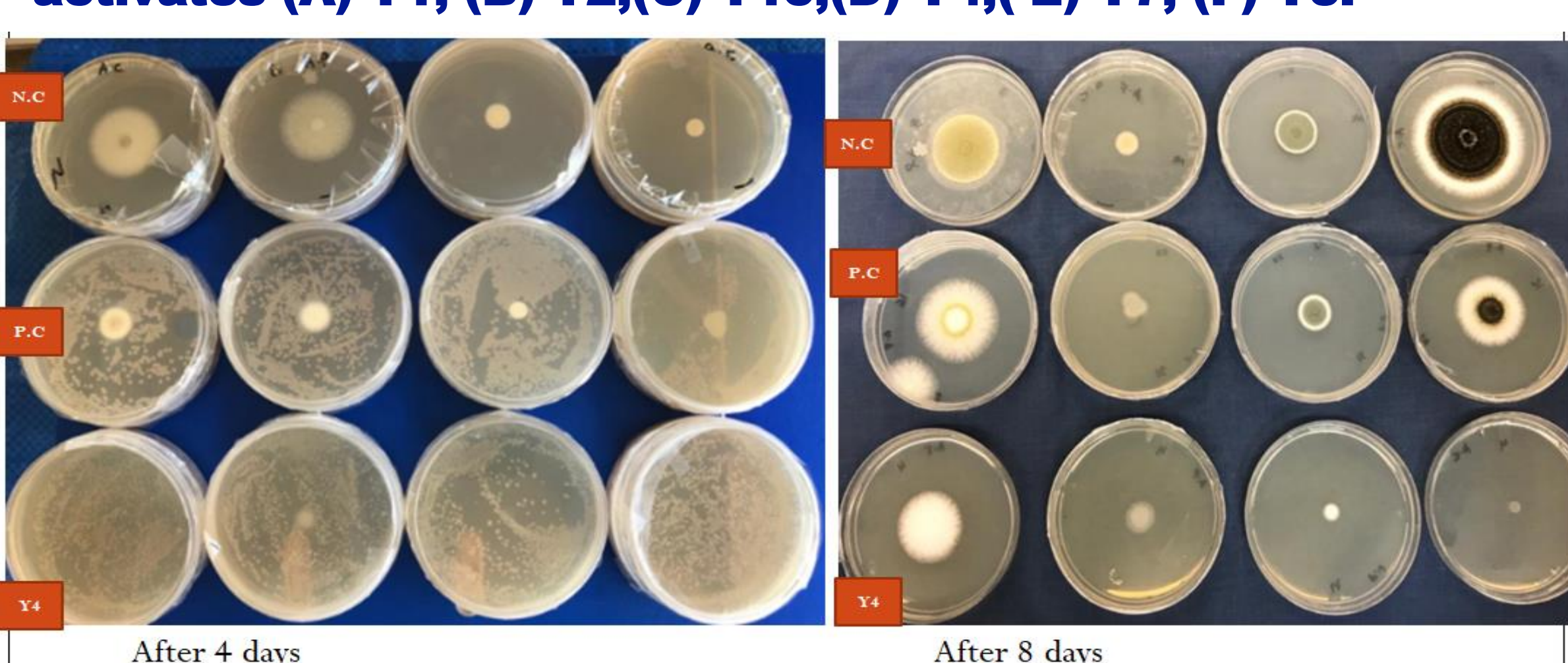

(A) shows the antifungal activities of yeast strain \#4 after 4

days of incubation.
( \& \& C ) Antifungal activities after 8 days incubation.

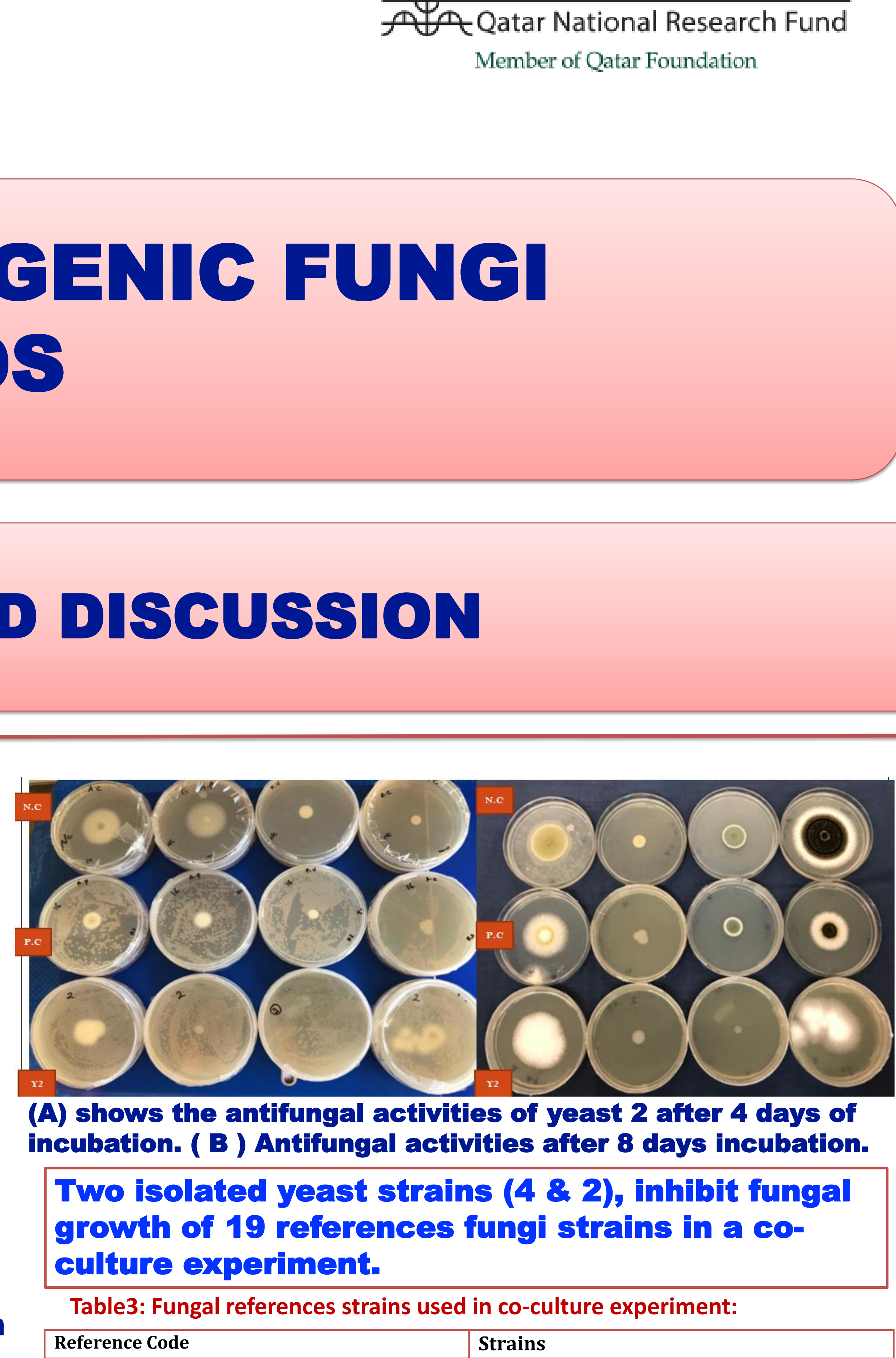

(A) shows the antifungal activities of yeast 2 after 4 days of Two isolated yeast strains (4 \& 2), inhibit fungal 19 references fungi strains in a coculture experiment.

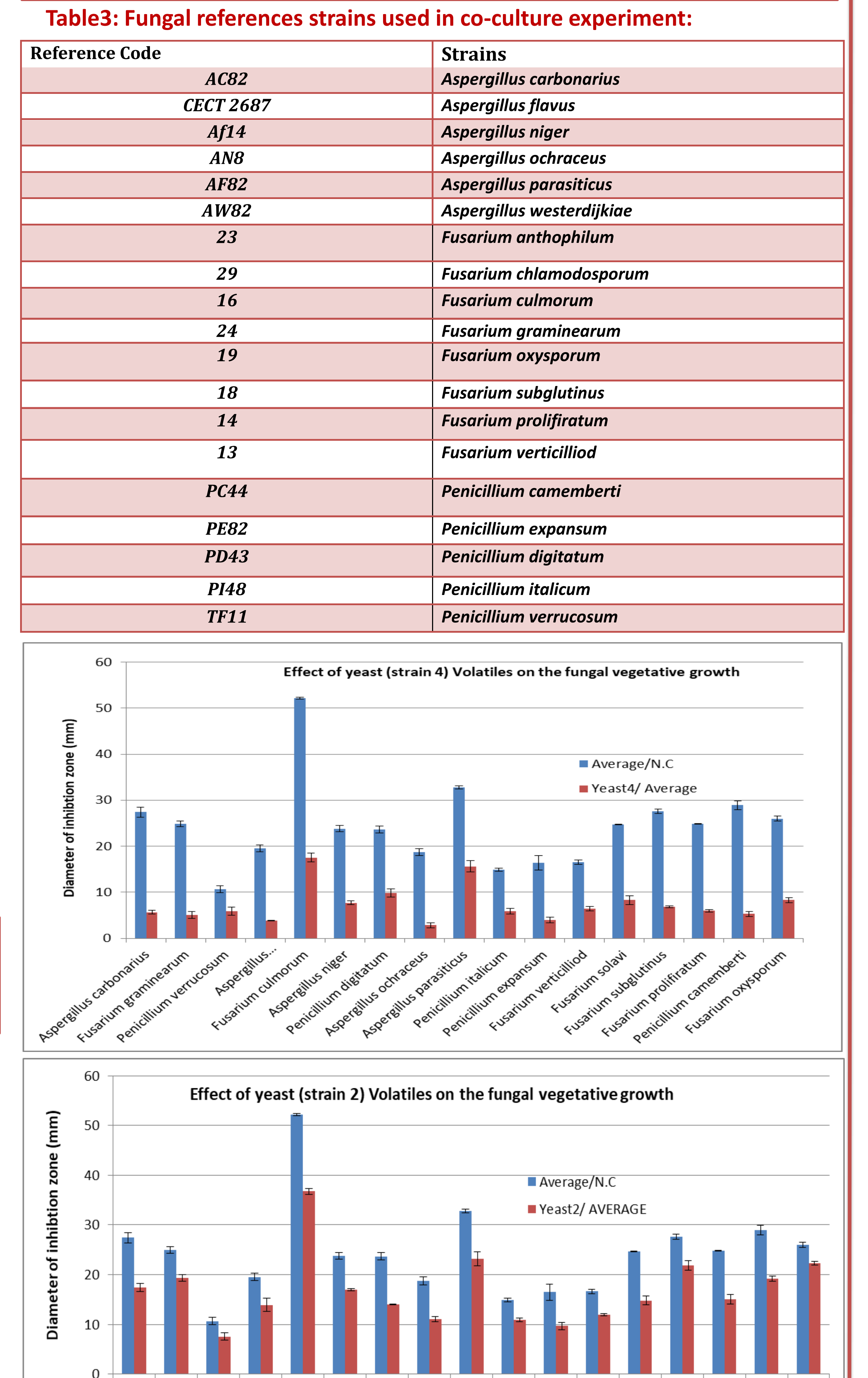

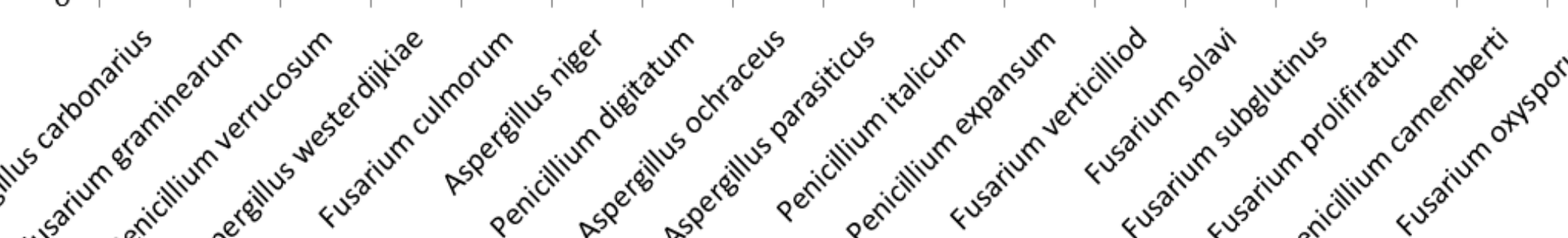

Diameters of antifungal growth of 19 fungal references strains against yeast strains ( 4 \& 2) in a co-culture

\section{CONCLUSION}

A total number of 15 fungal strains isolated from marketed Mixed dairy cow feed were tested for the AFB1 \& OTA synthesis.

Most of the fungi isolated synthesize high

concentrations of mycotoxins

A total of 15 yeast strains locally isolated from fermented food, meat and dairy products, we screened for antifungal activities against 20 fungi reference strains.

Yeast strains (4 \& 2) isolated from fermented food, have shown a great antifungal inhibition growth in-vitro as well as spores inhibition and mycotoxins synthesis.

The VOCs molecules are being identified

\section{Acknowledgments}

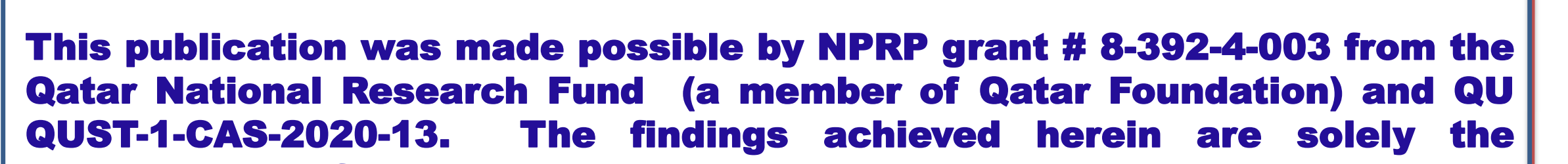
Special Thanks to Dr. Zahoor UI Hassan and Randa Zeidan
Sosponsibitit of the autho 